\title{
Some common fixed-point theorems for a pair of $p$-hybrid mappings via common limit range property in $G$-metric space
}

\author{
Lucas Wangwe ${ }^{a}$, Santosh Kumar ${ }^{a}$ \\ ${ }^{a}$ Department of Mathematics, College of Natural and Applied Sciences, University of Dar es Salaam, Dar es Salaam, Tanzania. .
}

\begin{abstract}
This paper aims to prove common fixed point theorems for a pair of hybrid and $p$-hybrid mappings via common limit range property in $G$-metric space setting. The theorems proved here will generalise the results due to Nashine et al. [35], Karapinar et al. 23, 24] from metric space notion to $G$-metric space and that of Mustafa et al.[30] using $\left(C L R_{f}\right)$ property concept in $G$-metric space, and many others in this setting. We also provided an illustrative example to validate the results.
\end{abstract}

Keywords: Common fixed point, $p$-hybrid mapping, common limit range property, $G$-metric space. 2010 MSC: 47H10, 54H25.

\section{Introduction}

In 1963, Gähler [12] introduced the notion of 2-metric space which made several other authors to use his notion to prove fixed point theorems for mappings on such spaces. A 2-metric is a function $d(x, y, z)$ symmetric under permutations, satisfying the tetrahedral inequality

$$
d(x, y, z) \leq d(x, y, a)+d(x, a, z)+d(a, y, z), \forall x, y, z, a \in X
$$

as well as conditions

(i) for all $x, y$, we have $d(x, y, y)=0$,

(ii) for all $x, y$, there exists $z$ such that $d(x, y, z) \neq 0$.

Email addresses: wangwelucas@gmail.com (Lucas Wangwe), drsengar2002@gmail.com (Santosh Kumar) 
Later on, many authors used his notion to generalise Banach's contraction principle to obtain fixed point theorems, e.g. [11, 19, 21, 25, 26, 41, 51] and many others. In 1988, Ha et al. [15] showed that a 2-metric need not be a continuous function of its variables, whereas an ordinary metric is a continuous function of its variables. Further, there is no easy relationship between results obtained in the two settings. In particular, the contraction mapping theorem in metric spaces and 2-metric spaces are unrelated. These considerations motivated Dhage [8] to introduce a new class of generalised metric space called D-metric space which is as follows:

Definition 1.1. [8] A function $D: X \times X \times X \rightarrow \mathbb{R}$ is said to be a $D$-metric on $X$ if it satisfies the following properties.

(i) $D(x, y, z) \geq 0$ for all $x, y, z \in X$ and equality holds if and only if $x=y=z$ (nonnegativity)

(ii) $D(x, y, z)=D(x, z, y)$ (symmetry)

(iii) $D(x, y, z) \leq D(x, y, a)+D(x, a, z)+D(a, y, z)$ for all $x, y, z \in X$ (tetrahedral inequality).

A nonempty set $X$ together with a $D$-metric is called a $D$-metric space and is denoted by $(X, D)$.

Using this definition, Dhage[8] proved the existence of a unique fixed point for self-mapping satisfying a contractive condition.

On the other hand, the existence of fixed points in $D$-metric space has been considered by Rhoades [40] to prove two fixed point theorems for the generalised metric spaces. Dhage [9, 10] proved the results for a common fixed point principle in $D$-metric space. Ahmad et al. [4] introduced a fixed point theorem for expansive mappings in $D$-metric space. Singh and Sharma [45] and Sedghi et al. [42] proved common fixed point theorems under compatible and weakly commuting mappings in $D$-metric space.

In 2006, Mustafa and Sims [29, 32] gave a generalisation of $D$-metric space to $G$-metric space soon after identifying some shortcomings concerning the fundamental topological structure on $D$-metric spaces. They defined several notions, such as continuity, completeness, compactness, convergence, and space product in the $G$-metric space setting. In doing so, they replaced the tetrahedral inequality with an inequality involving the repetition of indices. Further, Mustafa et al. [30, gave existence of a fixed point for mapping in $G$-metric spaces which influenced many other authors. Since then, several researchers proved fixed point theorems in $G$ metric space. Shushanta [47] proved property $P$ of Ciric operator on $G$-metric spaces. Chugh et al. [7] proved the results of property $P$ in $G$-metric spaces. Agarwal et al. [2] gave a theorem on couple fixed point results in asymmetric $G$-metric spaces. Rani et al. [39] introduced the version of common fixed point theorems for compatible and weakly compatible maps in $G$-metric spaces. Moreover, Jleli, and Samet [20] gave some remarks results on $G$-metric spaces. For more details on $G$-metric spaces, one can see $[3,5,28,31,33,43,44,49,50]$ and the reference therein.

Likewise, Sintunavarat and Kumam [46] introduced the notion of common limit range (CLR) property for single-valued mappings, which completely accommodated the conditions of the closeness of the degrees of the detailed mappings and showed its superiority over the (EA) property due to Aamri and El Moutawakil [1. Motivated by this fact, Imdad et al. [17] established a common limit range property for a hybrid pair of mappings and proved some fixed point results in symmetric (semi-metric) spaces. Also, Imdad et al. [16] established the joint common limit range notion and proved the common fixed point theorem for a pair of non-self mappings in metric space.

Some special conditions on the pairs of mappings like weakly compatible mappings, E.A. property, faintly compatible mappings, common limit range property $(C L R)$, coincidentally idempotent and joint common limit range property $(J C L R)$ has been utilised in different proofs by the researchers. One can see, [13, 16, 18, 36, 37, 38] and the references therein.

Naimpally et al. [34] generalised Goebel's [14 result in a hybrid of multivalued and single-valued maps satisfying a contractive condition. Consequently, several fixed point theorems for multivalued maps were extended by Naimpally et al. [34], Chauhan et al. [6] proved unified common fixed point theorems for a 
hybrid pair of mappings via an implicit relation involving altering distance function. Recently, Nashine et al. [35] established a prove on common fixed point theorem for hybrid generalised $(F, \psi)$-contraction under common limit range property in metric spaces.

Next, we will describe some preliminaries of definition and theorems which will be useful for developing our main results.

\section{Preliminaries}

Definition 2.1. [32] Let $X$ be a non empty set and $G: X \times X \times X \rightarrow \mathbb{R}_{+}$be a function satisfying the following conditions:

(G1) $G(x, y, z)=0$ if $x=y=z$;

(G2) $0<G(x, x, y)$, for all $x, y \in X$ with $z \neq y$;

(G3) $G(x, x, y) \leq G(x, y, z)$ for all $x, y \in X$ with $z \neq y$;

(G4) $G(x, y, z)=G(x, z, y)=G(y, z, x)=\ldots$ (symmetry in all three variables);

(G5) $G(x, y, z) \leq G(x, a, a)+G(a, y, z)$, for all $x, y, z, a \in X$ (rectangle inequality).

The function $G$ is called a generalised metric or $G$-metric, and the pair $(X, G)$ is called a $G$-metric space.

The following example satisfies the above axioms.

Example 2.2. [32] Let $X=\mathbb{R}$ be the set of all real number. Define $G: \mathbb{R}^{3} \rightarrow \mathbb{R}^{+}$by

$$
G(x, y, z)=|x-y|+|y-z|+|x-z|,
$$

for all $x, y, z \in X$. Then it is clear that $(X, G)$ is a $G$-metric space with a $G$-metric on $X$.

Note that if $G(x, y, z)=0$ then $x=y=z$.

Mustafa and Sims [32] proved the following proposition satisfying a $G$-metric properties.

Proposition 2.3. 32 Let $(X, G)$ be a $G$-metric space, then the metric associated with $G$ satisfies:

(i) $G(x, y, z) \leq G(x, x, y)+G(x, x, z)$,

(ii) $G(x, y, y) \leq 2 G(y, x, x)$,

(iii) $G(x, y, z) \leq G(x, a, a)+G(y, a, a)+G(z, a, a)$,

for all $x, y, z, a \in X$.

Mustafa and Sims [32] established some topological properties such as convergence, completeness and continuity in $G$-metric spaces as follows:

Definition 2.4. [32] Let $(X, G)$ be a $G$-metric space. A sequence $x_{n} \in X$ is said to be:

(i) $G$-convergent to $x \in X$ if for any $\epsilon>0$, there exists $k \in \mathbb{N}$ such that $G\left(x, x_{n}, x_{n}\right)<\epsilon$ for all $n \geq k$;

(ii) $G$-Cauchy if for $\epsilon>0$, there exists $k \in \mathbb{N}$ such that, for all $\left\{x_{n}\right\}$ in $X$ is called Cauchy sequence if for each $\epsilon>0$, there exists $n_{0} \in \mathbb{N}$ such that $G\left(x_{n}, x_{m}, x_{p}\right)<\epsilon$ for all $n, m, p \geq n_{0}$, i.e., $G\left(x_{n}, x_{m}, x_{p}\right) \rightarrow 0$ as $n, m, p \rightarrow \infty$. 
Definition 2.5. [32 A $G$-metric space is said to be $G$-complete if every $G$-Cauchy sequence in $X$ is $G$ convergent. Every $G$-metric on $X$ defines a metric

$$
d_{G}(x, y)=G(x, y, y)+G(y, x, x)
$$

for all $x, y \in X$.

Proposition 2.6. [32] Let $(X, G)$ be a $G$-metric space. Then the following properties are equivalent:

(i) $\left(x_{n}\right)$ is $G$-convergent to $x$,

(ii) $G\left(x_{n}, x_{m}, x_{n}\right) \rightarrow 0$ as $n, m \rightarrow \infty$,

(iii) $G\left(x_{n}, x, x\right) \rightarrow 0$ as $n \rightarrow \infty$,

(iv) $\left(x_{n}\right)$ is a $G$-Cauchy sequence,

(v) For every $\epsilon>0$, there exists $k \in \mathbb{N}$ such that $G\left(x_{n}, x_{m}, x_{m}\right)<\epsilon$ for $n, m>k$.

Definition 2.7. 32] Let $(X, G)$ and $\left(X^{\prime}, G^{\prime}\right)$ be two $G$-metric spaces and let $f:(X, G) \rightarrow\left(X^{\prime}, G^{\prime}\right)$. Then the map $f$ is said to be $G$-continuous at $x \in X$ if for $\epsilon>0$, there exists $\delta>0$ such that for all $x, y \in X$ and $G(a, x, y)<\delta$, we have $G^{\prime}(f a, f x, f y)<\epsilon$. The function $f$ is $G$-continuous if it is $G$-continuous for each $a \in X$.

Proposition 2.8. 32] Let $(X, G)$ and $\left(X^{\prime}, G^{\prime}\right)$ be two $G$-metric spaces and let $f:(X, G) \rightarrow\left(X^{\prime}, G^{\prime}\right)$. Then the map $f$ is said to be $G$-continuous at $x \in X$ if and only if $f$ is sequentially continuous, i.e., whenever $\left(x_{n}\right)$ is $G$-convergent to $x$, the sequence $f\left(x_{n}\right)$ is $G$-convergent to $f x$.

Lemma 2.9. 32] Let $(X, G)$ be a $G$-metric space. Then the function $G(x, y, z)$ is continuous in all it is variables.

Later, Kaewcharoen et al. [22] established the multi-valued notion in $G$-metric space. Let $X$ be a $G$ metric space. Denote $C B(X)$ be the class of all non-empty, closed and bounded subsets of $X$. Let $H_{G}(., .,$. be the Haursdorff- $G$-distance on $C B(X)$, that is for $A, B, C \in(C B(X))$ define:

$$
H_{G}(A, B, C)=\max \left\{\sup _{x \in A} G(x, B, C), \sup _{x \in B} G(x, C, A), \sup _{x \in C} G(x, A, B)\right\},
$$

where,

$$
\begin{aligned}
G(x, B, C) & =d_{G}(x, B)+d_{G}(B, C)+d_{G}(x, C), \\
d_{G}(x, B) & =\inf \left\{d_{G}(x, y), y \in B\right\}, \\
d_{G}(A, B) & =\inf \left\{d_{G}(a, b), a \in A, b \in B\right\}, \\
d_{G}(x, y, C) & =\inf \{G(x, y, z), z \in C\} .
\end{aligned}
$$

Lemma 2.10. 22] Let $X, G$ be a $G$-metric space and $A, B \in C B(X)$. Then for each $a \in A$, we have

$$
G(a, B, B) \leq H_{G}(A, B, B) .
$$

Lemma 2.11. [48] Let $(X, G)$ be a $G$-metric space and $A, B \in C B(X)$, then for each $\epsilon>0$, there exists $b \in B$ such that

$$
G(a, b, b) \leq H_{G}(A, B, B)+\epsilon .
$$


Definition 2.12. 22] Let $X$ be a non-empty set. Assume $f: X \rightarrow X$ and $T: X \rightarrow 2^{X}$ are two mappings. If $w=f x \in T x$ for some $x \in X$, then $x$ is called a coincidence point of the pair $(T, f)$ and $w$ is a point of coincidence of $f$ and $T$. The mapping $f$ and $T$ are called weakly compatible if $f x \in T x$ for some $x \in X$ implies $f T x \subseteq T f x$.

Proposition 2.13. 22] Let $X$ be a non-empty set. Assume $f: X \rightarrow X$ and $T: X \rightarrow 2^{X}$ are weakly compatible mappings. If $f$ and $T$ have a unique point of coincidence $u=f x \in T x$, then $u$ is a unique common fixed point of $f$ and $T$.

Imdad et al. [17, established the concept of common limit range property for a pair of hybrid mappings as follows.

Definition 2.14. [17] Let $(X, d)$ be a metric space with $f: X \rightarrow X$ and $T: X \rightarrow 2^{X}$ be single and multivalued mappings. Then the pair of hybrid mappings $(f, T)$ are said to have the $(C L R)$ property, If there exists a sequence $\left\{x_{n}\right\}$ in $X$ and $A \in 2^{X}$ such that

$$
\lim _{n \rightarrow \infty} f x_{n}=f u \in A=\lim _{n \rightarrow \infty} T x_{n},
$$

for some $u, v \in X$ and $A \in C B(X)$.

Mustafa et al. [27] defined a concept of 0- mappings on $G$-metric spaces as follows:

Definition 2.15. [27] Let $(X, d)$ be a $G$ - metric space and let $T: X \rightarrow X$ be a mapping. For $A \subset X$, let $\delta(A)=\sup \{G(a, b, c), a, b, c \in A\}$ and $\forall x, y \in X$, define,

$$
\begin{aligned}
0(x, T, n) & =\left\{x, T x, T^{2} x, \ldots T^{n} x\right\}, \\
0(x, T, \infty) & =\left\{x, T x, T^{2} x, T^{3} x \ldots\right\} .
\end{aligned}
$$

Definition 2.16. 27] Let $\left\{x_{n}\right\}_{n=0}^{\infty}$ be a sequence of elements of $X$, then for $i, j$, let

$$
\begin{aligned}
0\left(x_{i}, j\right) & =\left\{x_{i}, x_{i+1}, x_{i+2}, \ldots x_{i+j}\right\}, \\
0\left(x_{i}, \infty\right) & =\left\{x_{i}, x_{i+1}, x_{i+2}, \ldots\right\} .
\end{aligned}
$$

Mustafa et al. [27] proved their results on common fixed points in $G$-metric spaces using the concept of (E.A.) property as follows:

Theorem 2.17. [27] Let $(X, G)$ be a complete $G$ - metric space and suppose mapping $f, g: X \rightarrow X$ satisfy the following conditions:

(i) $f$ and $g$ are $G$-weakly commuting of type $G_{f}$,

(ii) $f(X) \subseteq g(X)$,

(iii) $g(X)$ is a $G$-complete subspace of $X$,

(iv) $G(f x, f y, f z) \leq \phi(M(x, y, z))$, for all $x, y, z \in X$,

where

$$
M(x, y, z) \leq\left\{\begin{array}{c}
G(g x, g y, g z), G(g x, f y, g x) \\
G(g y, f x, g y), G(g z, f x, g z) \\
G(g z, f y, g z), G(g y, g z, g y), \\
G(g x, f z, g x) .
\end{array}\right\}
$$

If there exists $x_{0} \in X$ such that $\delta\left(0\left(x_{0}, f, \infty\right)\right)<\infty$ then $f$ and $g$ have a unique common fixed point.

The notion of an almost altering distance function was introduced by Popa [36] which is as follows: 
Definition 2.18. [36] A function $\psi:[0, \infty) \rightarrow[0, \infty)$ is almost altering distance if

( $\Psi 1) \psi$ is continuous;

( $\Psi 2) \psi(t)=0$ if and only if $t=0$.

Example 2.19. [36]

$$
\psi(t)= \begin{cases}t, & \text { for } t=[0,1] ; \\ \frac{1}{t} & \text { for } t \in(1, \infty) .\end{cases}
$$

In 2020, Karapinar et al. 23] proved their results on $p$-hybrid Wardowski contraction as follows:

Definition 2.20. [23] A mapping $\mathcal{J}:(\mathcal{M}, d) \rightarrow(\mathcal{M}, d)$ is called a $p$-hybrid Wardowski contraction, if there is $G \in \beta$ such that

$$
d(\mathcal{J} v, \mathcal{J} w)>0 \Rightarrow \tau+G(d(\mathcal{J} v, \mathcal{J} w)) \leq G\left(\mathcal{A}_{\mathcal{J}}^{p}(v, w)\right)
$$

for all $p>0$. In particular, if the above inequality holds for $p=0$, we say the $\mathcal{J}$ is a 0 -hybrid Wardowski contraction.

Definition 2.21. 23, 24] Let $(\mathcal{M}, d)$ be a metric space and $\mathcal{J}$ be a self-mapping on this space for $p \geq 0$ and $k_{i} \geq 0, i=1,2,3,4$, such that $\sum_{i=1}^{4} k_{i}=1$, we define the following expression.

$$
\mathcal{A}_{\mathcal{J}}^{p}(v, w)=\left\{\begin{array}{l}
{\left[k_{1}(d(v, w))^{p}+k_{2}(d(v, \mathcal{J} v))^{p}+k_{3}(d(w, \mathcal{J} w))^{p}+k_{4}\left(\frac{d(v, \mathcal{J} v)+d(v, \mathcal{J} w)}{2}\right)^{p}\right]^{\frac{1}{p}}} \\
\text { for } p \geq 0, v, w \in \mathcal{M} \\
{[d(v, w)]^{K_{1}}[d(v, \mathcal{J} v)]^{K_{2}}[d(w, \mathcal{J} w)]^{K_{3}}\left[\frac{d(v, \mathcal{J} w)+d(w, \mathcal{J} v)}{2}\right]^{K_{4}},} \\
\text { for } p=0, v,\left.w \in \mathcal{M}\right|_{\mathcal{J}}(\mathcal{M}) .
\end{array}\right.
$$

Theorem 2.22. [Theorem 4 and 5] [23]

(1) A p-hybrid Wardowski contraction self mapping on a complete metric space admits exactly one fixed point in $\mathcal{M}$.

(2) A 0-hybrid Wardowski contraction self mapping on a complete metric space admits fixed point in $\mathcal{M}$ provided that for each sequence $\left\{n_{n}\right\}$ in $(0, \infty), \lim _{n \rightarrow \infty} n_{n}=0$ iff $\lim _{n \rightarrow \infty} G\left(n_{n}\right)=0$.

\section{Main Results}

To establish our first main result, we start by extending Definition 2.14 using the $G$-metric space concept.

Definition 3.1. Let $(X, G)$ be a $G$-metric space with $f: X \rightarrow X$ and $T: X \rightarrow C B(X)$ be single and multivalued mappings. Then the pair $(f, T)$ of hybrid mapping satisfy a common limit range $\left(C L R_{f}\right)$ property with respect to $f$, if there exists a sequence $\left\{x_{n}\right\}$ in $X$ and $A \in C B(X)$ such that

$$
\lim _{n \rightarrow \infty} f x_{n}=f u \in A=\lim _{n \rightarrow \infty} T x_{n},
$$

for some $u \in X$.

Now, we prove the following theorem which is an extension of Theorem 2.17

Theorem 3.2. Let $(X, G)$ be a complete $G$ - metric space and suppose mapping $f, T: X \rightarrow 2^{X}$ with almost altering distance $\psi \in \Psi$ satisfy the following conditions: 
(a) $f$ and $T$ are weakly compatible,

(b) $f$ and $T$ satisfy $C L R_{f}$ property,

(c) $T x \subseteq f(X)$,

(d) $T(X)$ is a $G$-complete subspace of $X$,

(e) $H_{G}(T x, T y, T z) \leq \psi(M(x, y, z))$; for all $x, y, z \in X$,

where

$$
M(x, y, z) \leq \max \left\{\begin{array}{c}
G(f x, f y, f z), G(f x, T y, f x), \\
G(f y, T x, f y), G(f z, T x, f z), \\
G(f z, T y, f z), G(f y, T z, f y), \\
G(f x, T z, f x)
\end{array}\right\} .
$$

Then $f$ and $T$ have a unique common fixed point.

Proof. From condition (a), using Definition 2.12 one can show that $f$ and $T$ are weakly compatible. Since $f x \in T x \Rightarrow f T x \subseteq T f x$. Thus $T(X) \subseteq f(X)$ and $T(X)$ is a $G$-complete subspace of $X$.

Applying Definition 3.1, as the pair $(f, T)$ satisfy $C L R_{f}$ property, there exists a sequence $\left\{x_{n}\right\}$ in $X$ such that

$$
\lim _{n \rightarrow \infty} f x_{n}=f u \in A=\lim _{n \rightarrow \infty} T x_{n},
$$

for some $u \in X$ and $A \in 2^{X}$.

First we show that $f u \in T u$. If $f u \neq T u$, for all $u \in X$, using Equation 7 with $x=x_{n}$ and $y=u$, we get

$$
H_{G}\left(T x_{n}, T u, T z\right) \leq \psi\left(M\left(x_{n}, u, z\right)\right),
$$

where

$$
M\left(x_{n}, u, u\right) \leq \max \left\{\begin{array}{c}
G\left(f x_{n}, f u, f z\right), G\left(f x_{n}, T u, f x_{n}\right), \\
G\left(f u, T x_{n}, f u\right), G\left(f z, T x_{n}, f z\right), \\
G(f z, T u, f z), G(f u, T z, f u), \\
G\left(f x_{n}, T z, f x_{n}\right)
\end{array}\right\}
$$

By taking $y=z$ in $(9)$ we have

$$
M\left(x_{n}, u, u\right) \leq \max \left\{\begin{array}{c}
G\left(f x_{n}, f u, f u\right), G\left(f x_{n}, T u, f x_{n}\right), \\
G\left(f u, T x_{n}, f u\right), G\left(f u, T x_{n}, f u\right), \\
G(f u, T u, f u), G(f u, T u, f u), \\
G\left(f x_{n}, T u, f x_{n}\right),
\end{array}\right\}
$$

Passing to the limits as $n \rightarrow \infty$ in (10) with $f x_{n}=f u, A=T x_{n}$, we obtain

$$
\begin{aligned}
M\left(x_{n}, u, u\right) & \leq \max \left\{\begin{array}{c}
G(f u, f u, f u), G(f u, A, f u), \\
G(f u, A, f u), G(f u, A, f u), \\
G(f u, A, f u), G(f u, A, f u), \\
G(f u, A, f u),
\end{array}\right\} \\
& \leq \max \left\{\begin{array}{c}
0, G(f u, A, f u), \\
G(f u, A, f u), G(f u, A, f u), \\
G(f u, A, f u), G(f u, A, f u), \\
G(f u, A, f u),
\end{array}\right\} \\
\Rightarrow M\left(x_{n}, u, u\right) & =G(f u, A, f u) .
\end{aligned}
$$


Using (11) in (8) as a results yields

$$
H_{G}(A, T u, T u) \leq \psi(G(f u, A, f u)) .
$$

As $\psi$ is non-decreasing we have

$$
H_{G}(A, T u, T u)<G(f u, A, f u),
$$

Using (13) and Lemma 2.11, we get

$$
\begin{aligned}
G(f u, T u, T u) & \leq G(f u, A, f u)+\epsilon, \\
G(f u, T u, T u)-G(f u, A, f u) & \leq \epsilon, \\
\Rightarrow 0 & <\epsilon,
\end{aligned}
$$

which is a contradiction. Hence, $f u \in T u$ which shows that the pair $(f, T)$ has a coincidence point.

Next, assume that if $f u \in T u$ and $f v \in T v$. By Lemma 2.10 and Proposition 2.13, we have

$$
G(f u, v, v)<G(T u, T v, T v) .
$$

Now, we prove the uniqueness of a point of coincidence of $f$ and $T$. We prove that the fixed point of $T$ is unique. Assume that $v \in X$ is another coincidence fixed point of $f$ and $T$ such that $v \neq u$. Then, since $u$ and $v$ are such that $f u \in T u, f v \in T v \in X$, we set $x=u$ and $y=v$ in (7) which yields

$$
H_{G}(T u, T v, T z) \leq \psi(M(u, v, z))
$$

where,

$$
M(u, v, z) \leq \max \left\{\begin{array}{c}
G(f u, f v, f z), G(f u, T v, f u), \\
G(f v, T u, f v), G(f z, T u, f z), \\
G(f z, T v, f z), G(f v, T z, f v), \\
G(f u, T z, f u)
\end{array}\right\} .
$$

By taking $y=z$ in 16 we have

$$
\begin{aligned}
& M(u, v, v) \leq \max \left\{\begin{array}{c}
G(f u, f v, f v), G(f u, T v, f u), \\
G(f v, T u, f v), G(f v, T u, f v), \\
G(f v, T v, f v), G(f v, T v, f v), \\
G(f u, T v, f u),
\end{array}\right\} \\
& \Rightarrow M(u, v, v)=G(f u, T v, f u) .
\end{aligned}
$$

Using (17) in (15) as a result yields

$$
H_{G}(T u, T v, T v) \leq \psi(G(f u, T v, f u)) .
$$

As $\psi$ is non-decreasing we have,

$$
H_{G}(T u, T v, T v)<G(f u, T v, f u),
$$

which is a contradiction. Hence, $f v \in T v$ which shows that the pair $(f, T)$ has a coincidence point. Thus, $(f, T)$ has a unique common fixed point which is $u$, that is the assumption given in Theorem 3.2 validated.

Furthermore, we formulate the corollaries:

Corollary 3.3. Let $(X, G)$ be a $G$-metric space. Assume that $f: X \rightarrow X$ and $T: X \rightarrow 2^{X}$ satisfy the conditions of Theorem 3.2 if any of the following contractions is applied: 
(i)

$$
H_{G}(T x, T y, T y)=k \max \left\{\begin{array}{c}
G(f y, T y, T y)+ \\
(G(f x, T y, T y), 2 G(f y, T x, T x))
\end{array}\right\}
$$

for all $x, y, z \in X$, where $k \in\left[0, \frac{1}{3}\right)$.

(ii)

$$
H_{G}(T x, T y, T z)=\left\{\begin{array}{c}
G(f x, f y, f z), G(f x, T x, T x) \\
G(f y, T y, T y), G(f y, T z, T z) \\
G(f x, T y, T y), G(f x, T z, T z), \\
G(f z, T x, T x),
\end{array}\right\}
$$

(iii)

$$
H_{G}(T x, T y, T z)=\left\{\begin{array}{c}
\alpha G(f x, f y, f z)+ \\
\beta[G(f x, T x, T x)+G(f y, T y, T y) \\
+G(f z, T z, T z)]
\end{array}\right\}
$$

for all $x, y, z \in X$ and $0 \leq \alpha+3 \beta<1$.

(iv)

$$
H_{G}(T x, T y, T z) \leq \psi(M(x, y, z))
$$

where

$$
M(x, y, z)=\max \left\{\begin{array}{c}
G(f x, f y, f y), G(f x, T x, T x) \\
G(f y, T y, T y), G(f z, T z, T z), \\
\frac{G(f x, f y, T x)}{2}, \\
\frac{G(f x, T y, T y)+G(f x, T z, T z)+G(f y, T x, T x)}{\frac{G(f x, f y, T x)}{2},} \\
\frac{G(f x, T y, T y)+G(f y, T x, T x)+G(f z, T x, T x)}{5}
\end{array}\right\}
$$

for all $x, y, z \in X$, where $k \in\left[0, \frac{1}{2}\right)$.

Corollary 3.4. Let $(X, G)$ be a $G$-metric space. Assume that $f: X \rightarrow X$ and $T: X \rightarrow 2^{X}$ be a pair of hybrid mappings. Then $(f, T)$ is called a generalized Meir-Keeler type contraction whenever for each $\epsilon>0$, there exists $\delta>0$ such that

$$
\epsilon \leq \psi(M(x, y, z))<\epsilon+\delta \Longrightarrow H_{G}(T x, T y, T z)<\epsilon,
$$

where

$$
M(x, y, z)=\max \left\{\begin{array}{l}
G(f x, f y, f z), G(T x, f x, f x) \\
G(T y, f y, f y), G(T z, f z, f z)
\end{array}\right\}
$$

for all $x, y, z \in X$. Then $(f, T)$ has a unique common fixed point. 
Next, we present our second main results, by extending Definition 2.21 using a pair of $p$-hybrid mapping in $G$-metric space concept.

Definition 3.5. Let $(X, G)$ be a $G$-metric space and $f, T$ be a pair of hybrid mapping on this space for $p \geq 0$ and $k_{i} \geq 0, i=1,2,3,4$, such that $\sum_{i=1}^{4} k_{i}=1$. We define the following expression.

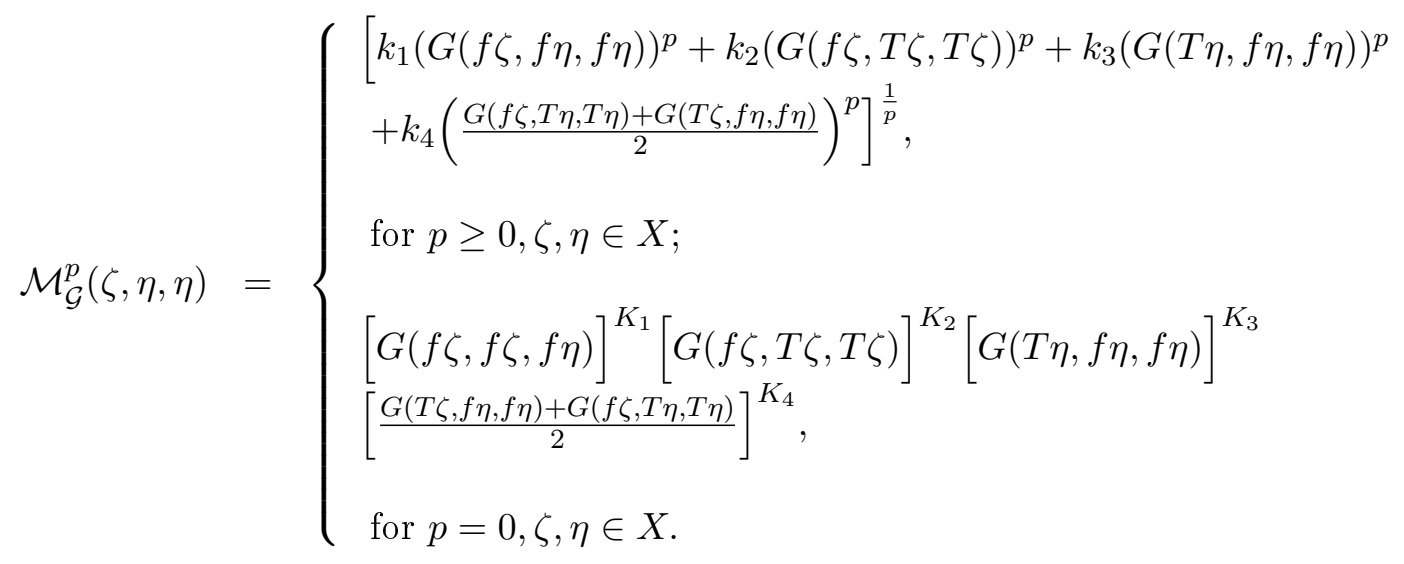

Theorem 3.6. Let $(X, G)$ be a complete $G$ - metric space and suppose mapping $f, T: X \rightarrow C B(X)$ is a $p$-hybrid contraction with almost altering distance $\psi \in \Psi$ satisfy the following conditions:

(a) $f$ and $T$ are weakly compatible;

(b) $f$ and $T$ satisfy $C L R_{f}$ property;

(c) $T x \subseteq f(X)$;

(d) $T(X)$ is a $G$-complete subspace of $X$;

(e) $\mathcal{H}_{G}^{p}(T \zeta, T \eta, T \eta) \leq \psi\left(\mathcal{M}_{\mathcal{G}}^{p}(\zeta, \eta, \eta)\right)$; for all $\zeta, \eta \in X$ and $p \geq 0$,

where

$$
\begin{aligned}
\mathcal{M}_{\mathcal{G}}^{p}(\zeta, \eta, \eta)= & {\left[k_{1}(G(f \zeta, f \eta, f \eta))^{p}+k_{2}(G(f \zeta, T \zeta, T \zeta))^{p}+k_{3}(G(T \eta, f \eta, f \eta))^{p}\right.} \\
& \left.+k_{4}\left(\frac{G(f \zeta, T \eta, T \eta)+d(T \zeta, f \eta, f \eta)}{2}\right)^{p}\right]^{\frac{1}{p}}
\end{aligned}
$$

Then $f$ and $T$ admit a unique common fixed point in $X$.

Proof. Applying Definition 3.1, as the pair $(f, T)$ satisfy $C L R_{f}$ property, there exists a sequence $\left\{x_{n}\right\}$ in $X$ such that

$$
\lim _{n \rightarrow \infty} f \zeta_{n}=f w \in B=\lim _{n \rightarrow \infty} T \zeta_{n},
$$

for some $w \in X$ and $B \in C B(X)$.

We assume that $f w \in T w$. If $f w \neq T w$, for all $w \in X$, using (26) with $\zeta=\zeta_{n}$ and $\eta=w$, we have

$$
\begin{aligned}
H_{G}^{p}\left(T \zeta_{n}, T w, T w\right) \leq & \leq\left(\mathcal{M}_{\mathcal{G}}^{p}\left(\zeta_{n}, w, w\right)\right), \\
\mathcal{M}_{\mathcal{G}}^{p}\left(\zeta_{n}, w, w\right)= & {\left[k_{1}\left(G\left(f \zeta_{n}, f w, f w\right)\right)^{p}+k_{2}\left(G\left(f \zeta_{n}, T \zeta_{n}, T \zeta_{n}\right)\right)^{p}+k_{3}(G(T w, f w, f w))^{p}\right.} \\
& \left.+k_{4}\left(\frac{G\left(f \zeta_{n}, T w, T w\right)+G\left(T \zeta_{n}, f w, f w\right)}{2}\right)^{p}\right]^{\frac{1}{p}}
\end{aligned}
$$


Passing to the limit as $n \rightarrow \infty$ in (28) with $f \zeta_{n}=f w, B=T \zeta_{n}=T w$, we obtain

$$
\begin{aligned}
\mathcal{M}_{\mathcal{G}}^{p}\left(\zeta_{n}, w, w\right) \leq & {\left[k_{1}(G(f w, f w, f w))^{p}+k_{2}(G(f w, T w, T w))^{p}+k_{3}(G(T w, f w, f w))^{p}\right.} \\
& \left.+k_{4}\left(\frac{G(f w, T w, T w)+G(T w, f w, f w)}{2}\right)^{p}\right]^{\frac{1}{p}}, \\
\leq & {\left[\left(k_{2}+k_{3}+k_{4}\right)(G(f w, T w, T w))^{p}\right]^{\frac{1}{p}} } \\
= & \left(k_{2}+k_{3}+k_{4}\right)^{\frac{1}{p}} G(f w, T w, T w) .
\end{aligned}
$$

Using (29) in (27) as a result yields

$$
H_{G}^{p}(B, T u, T u) \leq \psi\left(\left(k_{2}+k_{3}+k_{4}\right)^{\frac{1}{p}} G(f w, T w, T w)\right) .
$$

By the property of $\psi$, it follows that

$$
H_{G}^{p}(B, T w, T w)<G(f w, T w, T w) .
$$

Using (31) and Lemma 2.11, we get

$$
\begin{aligned}
G(f w, T w, T w) & \leq G(f w, T w, T w)+\epsilon, \\
G(f w, T w, T w)-G(f w, T w, T w) & \leq \epsilon \\
\Rightarrow 0 & <\epsilon
\end{aligned}
$$

which is a contradiction. Hence, $f w \in T w$ which shows that the pair $(f, T)$ has a coincidence point.

Next, assume that if $f w \in T w$ and $f z \in T z$. By Lemma 2.10 and Proposition 2.13, we have

$$
G(f z, z, z)<G(T z, T z, T z) .
$$

For the uniqueness point of coincidence of $f$ and $T$, we follow a similar procedure as in the proof of Theorem 3.2 .

Theorem 3.7. Let $(X, G)$ be a complete $G$ - metric space and suppose mapping $f, T: X \rightarrow C B(X)$ is a 0 -hybrid contraction with almost altering distance $\psi \in \Psi$ satisfy the following conditions:

(a) $f$ and $T$ satisfy $C L R_{f}$ property;

(b) $\mathcal{H}_{G}^{p}(T \zeta, T \eta, T \eta) \leq \psi\left(\mathcal{M}_{\mathcal{G}}^{p}(\zeta, \eta, \eta)\right)$; for all $\zeta, \eta \in X$ and $p=0$ with $k_{1}+k_{2}+k_{3}+k_{4}<1$, where

$$
\begin{aligned}
\mathcal{M}_{\mathcal{G}}^{p}(\zeta, \eta, \eta)= & {[G(f \zeta, f \zeta, f \eta)]^{K_{1}}[G(f \zeta, T \zeta, T \zeta)]^{K_{2}}[G(T \eta, f \eta, f \eta)]^{K_{3}} } \\
& {\left[\frac{G(T \zeta, f \eta, f \eta)+G(f \zeta, T \eta, T \eta)}{2}\right]^{K_{4}}, }
\end{aligned}
$$

Then $f$ and $T$ admits a unique common fixed point in $X$.

Proof. By Definition 3.1. $(f, T)$ satisfy $C L R_{f}$ property, there exists a sequence $\left\{x_{n}\right\}$ in $X$ such that

$$
\lim _{n \rightarrow \infty} f \zeta_{n}=f w \in B=\lim _{n \rightarrow \infty} T \zeta_{n},
$$

for some $w \in X$ and $B \in C B(X)$.

Suppose that $f w \in T w$. If $f w \neq T w$, for all $w \in X$, using 25$)$ with $\zeta=\zeta_{n}$ and $\eta=w$, we have

$$
\begin{aligned}
& H_{G}^{p}\left(T \zeta_{n}, T w, T w\right) \leq \psi\left(M\left(\zeta_{n}, w, w\right)\right), \\
& \mathcal{M}_{\mathcal{G}}^{p}\left(\zeta_{n}, w, w\right)=\left[G\left(f \zeta_{n}, f \zeta_{n}, f w\right)\right]^{K_{1}}\left[G\left(f \zeta_{n}, T \zeta_{n}, T \zeta_{n}\right)\right]^{K_{2}}[G(T w, f w, f w)]^{K_{3}} \\
& {\left[\frac{G\left(T \zeta_{n}, f w, f w\right)+G\left(f \zeta_{n}, T w, T w\right)}{2}\right]^{K_{4}} . }
\end{aligned}
$$


Passing to the limit as $n \rightarrow \infty$ in (35) with $f \zeta_{n}=f w, B=T \zeta_{n}=T w$, we obtain

$$
\begin{aligned}
\mathcal{M}_{\mathcal{G}}^{p}\left(\zeta_{n}, w, w\right) \leq & {[G(f w, f w, f w)]^{K_{1}}[G(f w, T w, T w)]^{K_{2}}[G(T w, f w, f w)]^{K_{3}} } \\
& {\left[\frac{G(T w, f w, f w)+G(f w, T w, T w)}{2}\right]^{K_{4}}, } \\
= & {[G(f w, T w, T w)]^{\left(k_{2}+k_{3}+k_{4}\right)} . }
\end{aligned}
$$

Using (36) in (34) as a result yields

$$
H_{G}^{p}(B, T u, T u) \leq \psi\left([G(f w, T w, T w)]^{\left(k_{2}+k_{3}+k_{4}\right)}\right) .
$$

By the property of $\psi$, it follows that

$$
H_{G}^{p}(B, T w, T w)<[G(f w, T w, T w)]^{\left(k_{2}+k_{3}+k_{4}\right)} .
$$

Using (38) and Lemma 2.11, we get

$$
\begin{aligned}
G(f w, T w, T w) & \leq[G(f w, T w, T w)]^{\left(k_{2}+k_{3}+k_{4}\right)}+\epsilon, \\
G(f w, T w, T w)-[G(f w, T w, T w)]^{\left(k_{2}+k_{3}+k_{4}\right)} & \leq \epsilon, \\
\Longrightarrow 0 & <\epsilon,
\end{aligned}
$$

which is a contradiction. Hence, $f w \in T w$ which shows that the pair $(f, T)$ has a coincidence point. From the proof of Theorem 3.2 , we conclude that $T$ is 0 -hybrid contraction in $G$-metric space. Also satisfies all conditions of Theorem 3.7 .

In the following section, we formulate a vivid example to validate Theorem 3.2 .

Example 3.8. Let $X=[0,1]$ be equipped with $G$-metric, $G(x, y, z)=|x-y|+|y-z|+|x-z|$. Define $T x=\left[0, \frac{x}{8(x+1)}\right], f x=x^{\frac{3}{2}}$ and $x_{n}=\frac{1}{n}$. Also, $\psi=\frac{1}{2} t, t>0$ for all $x, y, z \in X$.

Using Definition 2.12, the mappings $f$ and $T$ are called weakly compatible if $f x \in T x$ for some $x \in X$ which implies that $f T x \subseteq T f x$. To observe this consider, $T x=\left[0, \frac{x}{8(x+1)}\right]$ and $f x=x^{\frac{3}{2}}$.

For

$$
f T x=\left[\frac{x}{8(x+1)}\right]^{\frac{3}{2}},
$$

and

$$
T f x=\left[0, \frac{x^{\frac{3}{2}}}{8\left(x^{\frac{3}{2}}+1\right)}\right],
$$

so that

$$
\left[\frac{x}{8(x+1)}\right]^{\frac{3}{2}} \subseteq\left[0, \frac{x^{\frac{3}{2}}}{8\left(x^{\frac{3}{2}}+1\right)}\right] .
$$

Also, $f$ and $T$ satisfy $C L R_{f}$ property. By Definition 3.1 and let $\left\{x_{n}\right\}$ be a sequence in $X$. Then

$$
\begin{aligned}
& \lim _{n \rightarrow \infty} f\left(\frac{1}{n}\right)=f u \in A=\lim _{n \rightarrow \infty} T\left(\frac{1}{n}\right), \\
& \lim _{n \rightarrow \infty} f\left(\frac{1}{n}\right)=f 0 \in A=\lim _{n \rightarrow \infty} T\left(\frac{1}{n}\right) .
\end{aligned}
$$

Next, we show that $T X$ is a $G$-complete subspace in $X$. By Definition 2.5 we have

$$
\begin{aligned}
G(x, y, y) & \leq|x-y|+|y-y|+|x-y| \\
& =2|x-y|,
\end{aligned}
$$


and

$$
\begin{aligned}
G(y, x, x) & \leq|y-x|+|x-x|+|y-x| \\
& =2|y-x|
\end{aligned}
$$

Using (40) and (40) in (1), we get

$$
\begin{aligned}
d_{G}(x, y) & \leq 2|x-y|+2|y-x| \\
& =4|x-y| .
\end{aligned}
$$

To prove $(e)$, let $x, y, z \in X$. If $x=y=z=0$, then $T x=T y=T z=0$ and $H_{G}(T x, T y, T z)=0$. Our proof is done. If not suppose that the value of $x, y, z$ are not all zero.

For $x \leq y \leq z$, we have

$$
H_{G}(T x, T y, T z)=H_{G}\left(\left[0, \frac{x}{8(x+1)}\right],\left[0, \frac{y}{8(y+1)}\right],\left[0, \frac{z}{8(z+1)}\right]\right) .
$$

By (2)

$$
\Longrightarrow \max \left\{\begin{array}{l}
\sup _{0 \leq a \leq \frac{x}{8(x+1)}} G\left(a,\left[0, \frac{y}{8(y+1)}\right],\left[0, \frac{z}{8(z+1)}\right]\right), \\
\sup _{0 \leq b \leq \frac{y}{8(y+1)}} G\left(b,\left[0, \frac{x}{8(x+1)}\right],\left[0, \frac{z}{8(z+1)}\right]\right), \\
\sup _{0 \leq c \leq \frac{z}{8(z+1)}} G\left(a,\left[0, \frac{x}{8(x+1)}\right],\left[0, \frac{y}{8(y+1)}\right]\right)
\end{array}\right\} .
$$

Since $x \leq y \leq z$, then $\left[0, \frac{x}{8(x+1)}\right] \subseteq\left[0, \frac{y}{8(y+1)}\right] \subseteq\left[0, \frac{z}{8(z+1)}\right]$, using $[3$ implies that

$$
\begin{aligned}
& d_{G}\left(\left[0, \frac{x}{8(x+1)}\right],\left[0, \frac{y}{8(y+1)}\right]\right)=0, \\
& d_{G}\left(\left[0, \frac{y}{8(y+1)}\right],\left[0, \frac{z}{8(z+1)}\right]\right)=0, \\
& d_{G}\left(\left[0, \frac{x}{8(x+1)}\right],\left[0, \frac{z}{8(z+1)}\right]\right)=0 .
\end{aligned}
$$

Now for each $0 \leq a \leq \frac{x}{8(x+1)}$ and $d_{G}(x, y)=4|x-y|$ in $(3)$ we have 


$$
\begin{aligned}
G\left(a,\left[0, \frac{y}{8(y+1)}\right],\left[0, \frac{z}{8(z+1)}\right]\right)= & d_{G}\left(a,\left[0, \frac{y}{8(y+1)}\right]\right)+ \\
& d_{G}\left(\left[0, \frac{y}{8(y+1)}\right],\left[0, \frac{z}{8(z+1)}\right]\right)+ \\
& d_{G}\left(a,\left[0, \frac{z}{8(z+1)}\right]\right)=0 \\
\leq & 4 a-\frac{4 y}{8(y+1)}+0+4 a-\frac{4 z}{8(z+1)}, \\
= & 8 a-\frac{4 y(z+1)+4 z(y+1)}{8(y+1)(z+1)}, \\
\leq & \frac{8 x}{8(x+1)}-\frac{4 y(z+1)+4 z(y+1)}{8(y+1)(z+1)}, \\
= & \frac{8 x(y+1)(z+1)-(4 y(z+1)+4 z(y+1))(x+1)}{8(x+1)(y+1)(z+1)}
\end{aligned}
$$

Next, for each $0 \leq b \leq \frac{y}{8(y+1)}$ and $d_{G}(x, y)=4|x-y|$ in $(3)$ we have

$$
\begin{aligned}
G\left(b,\left[0, \frac{x}{8(x+1)}\right],\left[0, \frac{z}{8(z+1)}\right]\right)= & d_{G}\left(b,\left[0, \frac{x}{8(x+1)}\right]\right)+ \\
& d_{G}\left(\left[0, \frac{x}{8(x+1)}\right],\left[0, \frac{z}{8(z+1)}\right]\right)+ \\
& d_{G}\left(b,\left[0, \frac{z}{8(z+1)}\right]\right), \\
\leq & 4 b-\frac{4 x}{8(x+1)}+0+4 b-\frac{4 z}{8(z+1)}, \\
= & 8 b-\frac{4 x(z+1)+4 z(x+1)}{8(x+1)(z+1)}, \\
\leq & \frac{8 y}{8(y+1)}-\frac{4 x(z+1)+4 z(x+1)}{8(x+1)(z+1)}, \\
= & \frac{8 y(x+1)(z+1)-(4 x(z+1)+4 z(x+1))(y+1)}{8(x+1)(y+1)(z+1)}
\end{aligned}
$$


Similarly, for each $0 \leq c \leq \frac{z}{8(z+1)}$ and $(3)$ gives

$$
\begin{aligned}
G\left(c,\left[0, \frac{x}{8(x+1)}\right],\left[0, \frac{y}{8(y+1)}\right]\right)= & d_{G}\left(c,\left[0, \frac{x}{8(x+1)}\right]\right)+ \\
& d_{G}\left(\left[\frac{x}{8(x+1)}\right],\left[0, \frac{y}{8(y+1)}\right]\right)+ \\
& d_{G}\left(c,\left[0, \frac{y}{8(y+1)}\right]\right), \\
\leq & 4 c-\frac{4 x}{8(x+1)}+0+4 c-\frac{4 y}{8(y+1)}, \\
= & 8 c-\frac{4 x(y+1)+4 y(x+1)}{8(x+1)(y+1)}, \\
\leq & \frac{8 z}{8(z+1)}-\frac{4 x(y+1)+4 y(x+1)}{8(x+1)(y+1)}, \\
= & \frac{8 z(x+1)(y+1)-(4 x(y+1)+4 y(x+1))(z+1)}{8(x+1)(y+1)(z+1)}
\end{aligned}
$$

As a result, we deduce

$$
\begin{aligned}
H_{G}(T x, T y, T z) & \leq \max \left\{\begin{array}{l}
\sup _{0 \leq a \leq \frac{x}{8(x+1)}} \frac{8 x(y+1)(z+1)-(4 y(z+1)+4 z(y+1))(x+1)}{8(x+1)(y+1)(z+1)}, \\
\sup _{0 \leq b \leq \frac{y}{8(y+1)}} \frac{8 y(x+1)(z+1)-(4 x(z+1)+4 z(x+1))(y+1)}{8(x+1)(y+1)(z+1)}, \\
\sup _{0 \leq c \leq \frac{z}{8(z+1)}} \frac{8 z(x+1)(y+1)-(4 x(y+1)+4 y(x+1))(z+1)}{8(x+1)(y+1)(z+1)}
\end{array}\right\}, \\
& =\frac{8 z(x+1)(y+1)-(4 x(y+1)+4 y(x+1))(z+1)}{8(x+1)(y+1)(z+1)} .
\end{aligned}
$$


On the other hand, we calculate the following $G$-metrics. By (4) and (5), we get

$$
\begin{aligned}
& G(f x, f y, f z)=G\left(x^{\frac{3}{2}}, y^{\frac{3}{2}}, z^{\frac{3}{2}}\right) \\
& \leq\left|x^{\frac{3}{2}}-y^{\frac{3}{2}}\right|+\left|y^{\frac{3}{2}}-z^{\frac{3}{2}}\right|+\left|x^{\frac{3}{2}}-z^{\frac{3}{2}}\right| \text {, } \\
& =2 x^{\frac{3}{2}}-2 z^{\frac{3}{2}} \text {. } \\
& G(f x, T y, f x)=G\left(x^{\frac{3}{2}},\left[0, \frac{y}{8(y+1)}\right], x^{\frac{3}{2}}\right), \\
& \leq \inf \left\{d_{G}\left(x^{\frac{3}{2}}, 0\right), d_{G}\left(x^{\frac{3}{2}}, \frac{y}{8(y+1)}\right)\right\}, \\
& \left.\leq \inf \left\{4 x^{\frac{3}{2}}, 4 x^{\frac{3}{2}}-\frac{4 y}{8(y+1)}\right)\right\} \text {, } \\
& =4 x^{\frac{3}{2}}-\frac{y}{8(y+1)} \text {. } \\
& G(f y, T x, f y)=G\left(y^{\frac{3}{2}},\left[0, \frac{4 x}{8(x+1)}\right], y^{\frac{3}{2}}\right), \\
& \leq \inf \left\{d_{G}\left(y^{\frac{3}{2}}, 0\right), d_{G}\left(y^{\frac{3}{2}}, \frac{x}{8(x+1)}\right)\right\}, \\
& \left.\leq \inf \left\{4 y^{\frac{3}{2}}, 4 y^{\frac{3}{2}}-\frac{x}{8(x+1)}\right)\right\} \text {, } \\
& =4 y^{\frac{3}{2}}-\frac{4 x}{8(x+1)} \text {. } \\
& G(f z, T y, f z)=G\left(z^{\frac{3}{2}},\left[0, \frac{y}{8(y+1)}\right], z^{\frac{3}{2}}\right), \\
& \leq \inf \left\{d_{G}\left(z^{\frac{3}{2}}, 0\right), d_{G}\left(z^{\frac{3}{2}}, \frac{4 y}{8(y+1)}\right)\right\}, \\
& \left.\leq \inf \left\{4 z^{\frac{3}{2}}, 4 z^{\frac{3}{2}}-\frac{4 y}{8(y+1)}\right)\right\} \text {, } \\
& =4 z^{\frac{3}{2}}-\frac{4 y}{8(y+1)} \text {. } \\
& G(f y, T z, f y)=G\left(y^{\frac{3}{2}},\left[0, \frac{z}{8(z+1)}\right], y^{\frac{3}{2}}\right), \\
& \leq \inf \left\{d_{G}\left(y^{\frac{3}{2}}, 0\right), d_{G}\left(y^{\frac{3}{2}}, \frac{z}{8(z+1)}\right)\right\}, \\
& \left.\leq \inf \left\{4 y^{\frac{3}{2}}, 4 y^{\frac{3}{2}}-\frac{4 z}{8(z+1)}\right)\right\} \text {, } \\
& =4 y^{\frac{3}{2}}-\frac{4 z}{8(z+1)} \text {. } \\
& G(f x, T z, f x)=G\left(x^{\frac{3}{2}},\left[0, \frac{z}{8(z+1)}\right], x^{\frac{3}{2}}\right), \\
& \leq \inf \left\{d_{G}\left(x^{\frac{3}{2}}, 0\right), d_{G}\left(x^{\frac{3}{2}}, \frac{z}{8(z+1)}\right)\right\}, \\
& \left.\leq \inf \left\{4 x^{\frac{3}{2}}, 4 x^{\frac{3}{2}}-\frac{4 z}{8(z+1)}\right)\right\}, \\
& =4 x^{\frac{3}{2}}-\frac{4 z}{8(z+1)} \text {. }
\end{aligned}
$$


Applying Equation (7) we obtain

$$
\begin{aligned}
M(x, y, z) & \leq \max \left\{\begin{array}{c}
2 x^{\frac{3}{2}}-2 z^{\frac{3}{2}}, 4 x^{\frac{3}{2}}-\frac{4 y}{8(y+1)}, 4 y^{\frac{3}{2}}-\frac{4 x}{8(x+1)}, 4 z^{\frac{3}{2}}-\frac{4 x}{8(x+1)}, \\
4 z^{\frac{3}{2}}-\frac{4 y}{8(y+1)}, 4 y^{\frac{3}{2}}-\frac{4 z}{8(z+1)}, 4 x^{\frac{3}{2}}-\frac{4 z}{8(z+1)},
\end{array}\right\} \\
& =4 z^{\frac{3}{2}}-\frac{4 y}{8(y+1)} .
\end{aligned}
$$

By (48) and (47) it follows that

$$
\frac{8 z(x+1)(y+1)-(4 x(y+1)+4 y(x+1))(z+1)}{8(x+1)(y+1)(z+1)} \leq \psi\left(4 z^{\frac{3}{2}}-\frac{4 y}{8(y+1)}\right) .
$$

This shows that all condition imposed in Theorem 3.6 are satisfied. Hence a pair of hybrid mapping $f$ and $T$ in $G$-metric space has a unique common fixed point $x=0$.

Acknowledgement: The authors are thankful to the learned reviewers for their valuable comments.

\section{References}

[1] M. Aamri, D. El Moutawakil, Some new common fixed point theorems under strict contractive conditions, J. Math. Anal. Appl. 270(1) (2002) 181-188.

[2] R.P. Agarwal, Z. Kadelburg, S. Radenović, On coupled fixed point results in asymmetric G-metric spaces, Journal of operators 2013(1) (2013).

[3] R.P. Agarwal, E. Karapınar, D. O'Regan, A. F. Roldan-Lopez-de-Hierro, Fixed point theory in metric type spaces, Fixed Point Theory and Applications, Switzerland: Springer (2015).

[4] B. Ahmad, M. Ashraf, B.E. Rhoades, Fixed point theorems for expansive mappings in D-metric spaces, Indian Journal of Pure and Applied Mathematics 32(10) (2001) 1513-1518.

[5] A. Azam, N. Mehmood, Fixed point theorems for multivalued mappings in G-cone metric spaces, Journal of Inequalities and Applications (1) (2013) 354.

[6] S. Chauhan, M.A. Khan, Z. Kadelburg, M. Imdad, Unified common fixed point theorems for a hybrid pair of mappings via an implicit relation involving altering distance function, Abstract and Applied Analysis Volume 2014, (2014) Article ID 718040 | https://doi.org/10.1155/2014/718040.

[7] R. Chugh, T. Kadian, A. Rani, B.E. Rhoades, Property P in G-metric spaces, Fixed Point Theory and Applications (2010) 201012 pages.

[8] B.C. Dhage, Generalised metric space and topological structure, I. Analele Atintifice ale Universitatii Al. I. Cuza din lasi. Serie Noua Mathematica 46(3) (2000) 24.

[9] B.C. Dhage, A common fixed point principle in D-metric spaces, Bulletin of the Calcutta Mathematical Society 91(6) (1999) 475-480.

[10] B.C. Dhage, A.M. Pathan, B.E. Rhoades, A general existence principle for fixed point theorems in D-metric spaces, International Journal of Mathematics and Mathematical Sciences 23(7) (2000) 441-448.

[11] B. Fisher, Mappings with a common fixed point, Math. Sem. Notes Kobe Univ. 7(1979) 115-148.

[12] S. Gähler, 2-metrische Räume und ihre topologische Struktur, Math. Nachr. (26)(1963) 115-148.

[13] V. Gupta, W. Shatanawi, A. Kanwar, Coupled Fixed Point Theorems Employing CLR-Property on V-Fuzzy Metric Spaces, Mathematics, 8(3) (2020), p.404.

[14] K. Goebel, Coincidence theorem, Bull. Acad. Polon. Sci. S6r. Sci. Math., 16 (1968) 733-735.

[15] K.S. Ha, Y.J. Cho, A. White, Strictly convex and 2-convex 2-normed spaces, Math. Japonica, 33(3) (1988) 375-384.

[16] M. Imdad, S. Chauhan, P. Kumam, Fixed point theorems for two hybrid pairs of non-self mappings under joint common limit range property in metric spaces, J. Nonlinear Convex Anal., 16 (2) ( 2015) 243-254.

[17] M. Imdad, S. Chauhan, A.H. Soliman, M.A. Ahmed, Hybrid fixed point theorems in symmetric spaces via common limit range property, Math., 47(4) (2014) 949-962.

[18] M. Imdad, S. Chauhan, Z. Kadelburg, Fixed point theorems for mappings with common limit range property satisfying generalised $(\psi, \phi)$-weak contractive conditions, Mathematical Sciences, 7(1) (2013) p.16.

[19] K. Iseki, Fixed point theorems in 2-metric spaces, Math. Semin. Notes, Kobe Univ., (3)(1975) $133-136$.

[20] M. Jleli, B. Samet, Remarks on G-metric spaces and fixed point theorems, Fixed Point Theory and Applications, 2012(1) (2012) p.210.

[21] G. Jungck, Commuting mappings and fixed points, American Mathematical Monthly, (3)(1978) 261-263.

[22] A. Kaewcharoen, A. Kaewkhao, Common fixed points for single-valued and multivalued mappings in G-metric spaces, Int. J. Math. Anal., 5 (2011) 1775-1790.

[23] E. Karapinar, H. Aydi, A. Fulga, On-Hybrid Wardowski Contractions, Journal of Mathematics, (2020) Article ID 1632526 8 pages. https://doi.org/10.1155/2020/1632526 
[24] E. Karapınar, O. Alqahtani, H. Aydi, On interpolative Hardy-Rogers type contractions, Symmetry 11(1) ( 2019$) 8$.

[25] M.S. Khan, On the convergence of sequences of fixed points in 2-metric spaces, Indian Journal of Pure and Applied Mathematics, 10(9) (1979) 1062-1067.

[26] T. Kubiak, Common fixed points of pairwise commuting mappings, Math. Nachr., (118), (1984) $123-127$.

[27] Z. Mustafa, H. Aydi, E. Karapınar, On common fixed points in G-metric spaces using (EA) property, Comput. Math. Appl., 64(6) (2012) 1944-1956.

[28] Z. Mustafa, H. Obiedat, A fixed points theorem of Reich in G-metric spaces, Cubo A Mathematics Journal, 12(1) (2010) 83-93.

[29] Z. Mustafa, B. Sims, Fixed point theorems for contractive mappings in complete G-metric spaces, Fixed Point Theory and Applications, (2009) 1-10.

[30] Z. Mustafa, W. Shatanawi, M. Bataineh, Existence of fixed point results in G-metric spaces, International Journal of Mathematics and Mathematical Sciences, (2009) 1-10.

[31] Z. Mustafa, M. Arshad, S.U. Khan, Ahmad, J. and Jaradat, MMM, Common fixed points for multivalued mappings in G-metric spaces, J. Nonlinear Sci. Appl., 10 (2017) 2550-2564.

[32] Z. Mustafa, B. Sims, A new approach to generalised metric spaces, Journal of Non-linear and convex Analysis, 7(2) (2006) 289-297.

[33] V. Nagaraju, Common Fixed Point Theorems for Six Self-Maps in G-metric spaces, Annals of Pure and Applied Mathematics, 22(1) (2020) 57-64.

[34] S.A. Naimpally, S.L. J. Singh, H.M. Whitfield Coincidence theorems for hybrid contractions, Math. Nachr., 127 (1986) 177-180.

[35] H. K. Nashine, M. Imdad, M. Ahmadullah, Common Fixed-Point Theorems for Hybrid Generalized $(F, \phi)$-Contractions Under the Common Limit Range Property with Applications, Ukrainian Mathematical Journal, 69(11) (2018) $1784-1804$.

[36] V. Popa, A.M. Patriciu, A general fixed point theorem for a pair of self mappings with common limit range property in $G$-metric spaces, Facta Universitatis, Series: Mathematics and Informatics, 29(4) (2015) 351-370.

[37] V. Popa, A.M. Patriciu, Fixed point theorems for two pairs of mappings satisfying common limit range property in $G$-metric spaces, Bul. Inst. Politeh. Iasi, Sect. I, Mat. Mec. Teor. Fiz, 62(66), (2016), 19-42.

[38] V. Popa, Fixed point theorems for two pairs of mappings satisfying a new type of common limit range property, Filomat, 31(11) (2017) 3181-3192.

[39] A. Rani, S. Kumar, N. Kumar, S.K. Garg, Common fixed point theorems for compatible and weakly compatible maps in $G$-metric spaces, Facta Universitatis, Series: Mathematics and Informatics, 2012(3) (2012) 1128-1134.

[40] B.E. Rhoades, A fixed point theorem for generalised metric spaces, Internat. J. Math. \& Math. Sci., 19(3) (1996) 457-460.

[41] B.E. Rhoades, Contraction type mappings on a 2-metric space, Math. Nachr., 91 (1979), 151-155.

[42] S. Sedghi, S. Nabi, Z. Haiyun, A common fixed point theorem in metric spaces, Fixed point theory and Applications, 2007 (2007) 13.

[43] W.A. Shatanawi, M. Abbas, Some fixed point results for multivalued mappings in ordered $G$-metric spaces, Gazi University Journal of Science, 25(2) (2012) 385-92.

[44] A. Shoaib, A. Shahzad, Common Fixed Point of Multivalued Mappings in Ordered Dislocated Quasi G-Metric Spaces, Punjab University Journal of Mathematics, 52(10) (2020).

[45] B. Singh, R.K. Sharma, Common fixed points via compatible maps in D-metric spaces, International Journal of Mathematics and Mathematical Sciences, 11(1) (2002) 145-153.

[46] W. Sintunavarat, P.Kumam, Common fixed point theorems for a pair of weakly compatible mappings in fuzzy metric spaces, J. Appl. Math., (2011) 1-14.

[47] K.M. Sushanta, Property P of Ciric operators in G-metric spaces, Inter. J. of Math. Sci. and Engg. Appls., 5(2) (2011) $353-367$.

[48] N. Tahat, H. Aydi, E. Karapınar, Shatanawi, W., Common fixed points for single-valued and multivalued maps satisfying a generalised contraction in G-metric spaces, Fixed Point Theory Appl., 10 (2012) 1-9.

[49] A. Tomar, J.C. Giniswamy, P. G. Maheshwari, Coincidence and common fixed point of F-contractions via CLRST property, Surv. Math. Appl., 11 (2016) 21-31.

[50] A. Tomar, R. Sharma, S. Upadhyay, Some applications of existence of common fixed and common stationary point of a hybrid pair, Bull. Int. Math. Virtual Inst. 9(1) (2018) 73-84.

[51] M. Khan, On fixed point theorems in 2-metric space, Publ. de l'Institute Math'ematique, 41 (1980), $107-112$. 